\title{
Low-Temperature Plasma Short-Exposure to Decontaminate Peri-Implantitis-Related Multispecies Biofilms on Titanium Surfaces
}

Beatriz H. D. Panariello

Indiana University School of Dentistry

Drashty P. Mody

Indiana University School of Dentistry

George J. Eckert

Indiana University School of Medicine

Paulo G. Coelho

New York University College of Dentistry

Simone Duarte ( $\nabla$ siduarte@iu.edu )

Indiana University School of Dentistry

\section{Research Article}

Keywords: Peri-implantitis, LTP, Actinomyces naeslundii, Biofilms, Fluorescence, CLSM

Posted Date: December 6th, 2021

DOI: https://doi.org/10.21203/rs.3.rs-1111970/v1

License: (c) (i) This work is licensed under a Creative Commons Attribution 4.0 International License. Read Full License 


\section{Abstract}

Peri-implantitis is a bacteria-initiated infection that as yet has no effective treatment. A novel approach to treat peri-implantitis is the use of low-temperature plasma (LTP). LTP disrupts the biofilm while conditioning the surrounding host environment for bone growth around the infected implant. The goal of this study was to evaluate the antimicrobial properties of LTP on newly formed (24-h) and mature (7days) peri-implant-related biofilms. Biofilm was composed of Actinomyces naes/undii (ATCC 12104), Porphyromonas gingivalis (W83), Streptococcus oralis (ATCC 35037), and Veillonella dispar (ATCC 17748). They were cultivated in brain heart infusion supplemented with $1 \%$ yeast extract, hemin $(0.5$ $\mathrm{mg} / \mathrm{mL})$, and menadione $(5 \mathrm{mg} / \mathrm{mL})$ and kept at 37区C in anaerobic conditions for 24-h. The species were mixed for a final concentration of $\sim 10^{5}$ colony forming units (CFU)/mL $(O D=0.01)$, and the bacterial suspension was transferred to 24 -well plates containing titanium specimens. Biofilms were treated with LTP for 1, 3, and $5 \mathrm{~min}$ at 3 or $10 \mathrm{~mm}$ from plasma-tip to sample. Controls were no treatment (Negative control=NC) and argon-flow at the same LTP conditions. Positive controls were $14 \mathrm{~g} / \mathrm{mL}$ amoxicillin and $140 \mu \mathrm{g} / \mathrm{mL}$ metronidazole individually or combined, and $0.12 \%$ chlorhexidine. Biofilms were evaluated by CFU, confocal laser scanning microscopy (CLSM), and Fluorescence in situ Hybridization (FISH). Wilcoxon Signed-Rank and Wilcoxon Rank Sum tests were applied $(a=0.05)$. Bacterial growth was observed in all no-treatment groups corroborated by FISH. LTP treatment significantly reduced all bacteria species when compared to the NC in both tested periods and in all treatment combinations $(p \leq 0.016)$, these results were corroborated by CLSM. There were no significant differences during biofilm development, between 24-h, 3, and 7 days within each LTP treatment, or among the bacteria within each LTP treatment $(p \geq 0.05)$. LTP application is effective to reduce peri-implantitis-related multispecies biofilms on titanium surfaces.

\section{Introduction}

The oral cavity microbiota impact biofilm development on newly placed implants. It is recognized that biofilm-induced inflammation around dental implants has a central role in stimulating osteoclastmediated bone resorption and hindering bone formation, causing net bone loss around implants [1]. This process is known as peri-implantitis, a bacteria-started infection that yet has no effective treatment [2]. Healthy and systemically compromised patients can develop peri-implantitis, which may result in the loss of their dental implants [3]. About $45 \%$ of patients that have dental implants will develop peri-implantitis in 10 years [4] [5].

It has been verified that the biofilm around an infected implant presents with a high occurrence of pathogens related to periodontitis [6], containing members of the red complex ( $P$. gingivalis, Treponema denticola, and Tannerella forsythia) and orange complex species (Fusobacterium sp. and Prevotella intermedia [7]. Common primary colonizers in the initial biofilm are streptococci, veillonellae, and actinomyces [8] [9] [10]. Streptococci and Actinomyces species can co-aggregate and offer attachment sites and growth support to other bacteria, such as Veillonellae spp., that have metabolic interactions with Streptococci [11]. Veillonella species can also grow mixed communities with diverse late colonizers 
[12]. The presence of $P$. gingivalis, which is known as a second colonizer [13], is related to periodontitis and these bacteria are often encountered at sites of peri-implantitis [14] [15] [16]. For the evaluation of anti-biofilm therapies, proper models are required, for example, multispecies biofilm models, to simulate the in vivo condition [17].

Biofilm organization of the peri-implant bacteria prevents disease elimination by conventional antimicrobial treatment because of their poor penetration into the biofilm, along with adverse effects of the antimicrobial treatment associated with bacterial resistance and systemic toxicity. Further challenging antimicrobial treatment of peri-implantitis is the need for the mechanical disruption of biofilms in intricate implant geometries that also present micrometer and nanometer-level textured surfaces. Since osteoblast adherence to surfaces is inhibited by endotoxins, detoxification of the surface would also improve implant integration [18]. Despite frequent use, there is a deficiency of data specifying the effects of antibiotic or other topical treatments on peri-implantitis-affected implant surfaces [19]. Therefore, a significant debate exists about the different properties of antimicrobial agents and to what extent these contribute to reducing peri-implantitis related biofilms.

Low-temperature plasma (LTP) has been documented as a powerful instrument for biomedicine and characterizes a new technology for the treatment of peri-implantitis [20]. Unlike current peri-implantitis treatment strategies, preliminary data demonstrate LTP's unique potential to simultaneously decontaminate and/or detoxify biofilm infected surfaces while allowing for the creation of a suitable environment for bone regrowth around the implant without damaging peri-implant healthy tissue [20-23]. LTP presents many advantages compared to traditional antimicrobial applications. It can be used for sitespecific treatment; it provides (i) a quick bactericidal response; (ii) antimicrobial resistance is less likely to occur because of LTP's multiple modes of action and diversity of active agents [21], and (iii) there are minimal side effects [21]. Even though translational model evidence is necessary to establish the safety of LTP, tissue-compatible temperatures of plasma encourage its in vivo use [22] [23].

LTP produces high responsiveness at low gas temperatures (under $40^{\circ} \mathrm{C}$ ). Electromagnetic radiation is produced, including ultra-violet radiation and light in the visible spectrum, and comprises excited gas particles, charged ions, free electrons, free radicals, neutral reactive oxygen, and nitrogen species [24]. Oxygen and nitrogen-based radicals are considered the most significant contributors to LTP sterilizing effects [21]. The reactive species have robust oxidative effects on the external structures of bacterial cells. Unsaturated fats in the lipid bilayer of cell membranes are susceptible to attack by hydroxyl radicals, compromising membrane function, while proteins in membranes are susceptible to oxidative damage by reactive species. As soon as the cell membranes have been in part degraded, the reactive species may damage genetic material and molecules within the bacteria, ultimately destroying it [25]. Furthermore, the antimicrobial dosage of LTP exhibited no sign of relevant damage on reconstituted oral epithelium (ROE) [23], and reconstituted gingival epithelium (RGE) [22], as demonstrated by low cytotoxicity and high cell viability levels [23]. In addition to the antimicrobial and tissue-friendly effects, multiple studies of LTP application on dental implant surfaces demonstrated that osseointegration 
increases up to $300 \%$ relative to non-treated implants resulting in superior implant biomechanical fixation in bone[26] [27] [28].

Given these advantages of LTP treatment, the present study aimed to evaluate in vitro LTP's antimicrobial activity against 24 h, 3, and 7 days multispecies per-implant related biofilms formed by Actinomyces naes/undii, P. gingivalis, Streptococcus oralis, and Veillonella dispar at different exposure times (1, 3 or 5 $\mathrm{min}$ ) and different distances from the equipment nozzle and the samples ( 3 or $10 \mathrm{~mm}$ ). We hypothesize that a single LTP treatment in vitro will be sufficient to disrupt and disinfect multispecies peri-implantitisrelated biofilm more effectively than the controls.

\section{Material And Methods}

\section{Bacterial strain and growth conditions}

Standard strains from the American Type Culture Collection (ATCC; Rockville, MD, USA) of Actinomyces naes/undii (ATCC 12104), Porphyromonas gingivalis (ATCC W83), Streptococcus oralis (ATCC 35037), and Veillonella dispar (ATCC 17748) were used to form the multispecies biofilms. Stock cultures were maintained at $-80^{\circ} \mathrm{C}$, reactivated onto $5 \%$ sheep blood agar plates (Dickinson and Company; Sparks, MD, USA) and incubated at $37^{\circ} \mathrm{C}$ for 7 days. After that, for the formation of the pre-inoculum, bacteria were individually reactivated by collecting colonies with the aid of a sterile loop and transferring them to a tube containing $5 \mathrm{~mL}$ of Brain Heart Infusion (BHI) broth culture medium (Acumedia; Lansing, MI, USA) supplemented with $1 \%$ yeast extract, hemin $(0.5 \mathrm{mg} / \mathrm{mL})$ and menadione $(5 \mathrm{mg} / \mathrm{mL})$ and kept at $37^{\circ} \mathrm{C}$ in anaerobic conditions $\left(80 \% \mathrm{~N}_{2}, 10 \% \mathrm{H}_{2}\right.$, and $\left.10 \% \mathrm{CO}_{2}\right)$ for $24 \mathrm{~h}$. For all microorganisms, co-cultures started from $\mathrm{OD}_{600 \mathrm{~nm}}=0.01[17]$.

\section{Biofilm formation}

Four-species biofilm was developed on the surfaces of titanium discs (Implacil De Bortoli; São Paulo, SP, Brazil). The discs sizes are $7.5 \mathrm{~mm}$ in diameter by $2 \mathrm{~mm}$ in thickness, with about $136 \mathrm{~mm}^{2}$ of total area. For multispecies biofilm formation, $A$. naes/undii, P. gingivalis, $S$. oralis, and $V$. disparwere mixed at the same ratio (1:1:1:1) at a final concentration of approximately $10^{5} \mathrm{CFU} / \mathrm{mL}\left(\mathrm{OD}_{600 \mathrm{~nm}}=0.01\right)$ and $1 \mathrm{~mL}$ of the multispecies bacterial suspension was transferred to each well containing a titanium specimen. The plates were kept at $37^{\circ} \mathrm{C}$ in anaerobic conditions for $24 \mathrm{~h}, 3$ days, and 7 days. For the 3 and 7 days biofilm formation, BHI broth culture medium supplemented with $1 \%$ yeast extract, hemin $(0.5 \mathrm{mg} / \mathrm{mL})$, and menadione $(5 \mathrm{mg} / \mathrm{mL})$ was replaced daily.

\section{Biofilm treatments and analysis}

After each period ( $24 \mathrm{~h}, 3$, and 7 days), the biofilms were treated with LTP (Plasma=P) for $1 \mathrm{~min}, 3 \mathrm{~min}$, and $5 \mathrm{~min}$, and $3 \mathrm{~mm}$ or $10 \mathrm{~mm}$ of distance between the plasma nozzle and the sample. Controls were: no treatment (Negative control=NC) and flow control (Flow= F) at the same conditions ( 1,3 or 5 min; 3 or $10 \mathrm{~mm}$ ). In addition, $14 \mathrm{~g} / \mathrm{mL}$ amoxicillin (AX) and $140 \mu \mathrm{g} / \mathrm{mL}$ metronidazole (MZ) individually or in 
combination, and $0.12 \%$ chlorhexidine (CHX) were used as positive controls (Figure 1). Biofilms formed on titanium discs were collected after treatments with a sterile micro spatula (Thermo Fisher Scientific; Waltham, MA, USA) and transferred to tubes containing $5 \mathrm{~mL}$ of $0.89 \% \mathrm{NaCl}$. Biofilms suspensions were sonicated (three $10 \mathrm{~s}$ pulses, $7 \mathrm{~W}$ output). An aliquot $(0.1 \mathrm{~mL})$ of the homogenized suspension was serially diluted and seeded onto $5 \%$ sheep blood agar blood plates (Dickinson and Company; Sparks, MD, USA). The plates were incubated at $37^{\circ} \mathrm{C}$ in anaerobic conditions for 7 days and then the $\mathrm{CFU} / \mathrm{mL}$ was calculated. Each bacteria was differentiated through their distinct morphology on the blood agar plates (Figure 2).

\section{Fluorescence in situ hybridization (FISH)}

FISH was performed according to Kommerein et al. [17] with few modifications. Biofilms from the NC of each period were rinsed once with Phosphate Buffered Saline (PBS) (Thermo Fisher Scientific; Waltham, MA, USA) and fixed using $2 \%$ glutaraldehyde (Thermo Fisher Scientific; Waltham, MA, USA) and a sequence of $50 \%, 80 \%$, and $99 \%$ ethanol. After drying, the fixed cells in the biofilms were permeabilized with $40 \mu \mathrm{L}$ of $1 \mu \mathrm{g} / \mu \mathrm{L}$ lysozyme for $15 \mathrm{~min}$ at $37^{\circ} \mathrm{C}$. Lysis was stopped by adding $200 \mu \mathrm{L}$ absolute ethanol for $3 \mathrm{~min}$, then, the samples were air-dried. A hundred microliters $(100 \mu \mathrm{L})$ of urea-NaCl buffer $(1$ $\mathrm{M}$ urea, $0.9 \mathrm{M} \mathrm{NaCl}, 20 \mu \mathrm{M}$ Tris-HCl; $\mathrm{pH}$ 7.0) together with $4 \mu \mathrm{L}$ of $100 \mu \mathrm{M}$ of each probe were applied to the biofilms (total $400 \mu \mathrm{L}$ of urea-NaCl buffer+4 probes). The applied probes were Alexa Fluor 488 for $A$. naes/undii (PMT detector; $488 \mathrm{~nm}$ laser), Alexa Fluor 647 for $P$. gingivalis (PMT detector; $638 \mathrm{~nm}$ laser), Alexa Fluor 405 for $S$. oralis (HyD detector; $405 \mathrm{~nm}$ laser), and Alexa Fluor 568 for $V$. dispar (HyD detector; $552 \mathrm{~nm}$ laser). Hybridization was made for $25 \mathrm{~min}$ at $46^{\circ} \mathrm{C}$. The biofilms were rinsed twice with $400 \mu \mathrm{L}$ of prewarmed urea-NaCl washing buffer (4 M urea, $0.9 \mathrm{M} \mathrm{NaCl}, 20 \mu \mathrm{M}$ Tris-HCl; pH 7.0) and then $400 \mu \mathrm{L}$ urea- $\mathrm{NaCl}$ washing buffer was applied to the biofilms and incubated for $5 \mathrm{~min}$ at $48^{\circ} \mathrm{C}$. After repeating this washing steps two more times, the biofilms were rinsed once with ultrapure water, then, $400 \mu \mathrm{L}$ of PBS was added, and subsequentially biofilms were visualized with a Leica SP8 DIVE confocal/2-photon microscope (Leica Microsystems, Buffalo Grove, IL) and a Leica HC PL APO CS2 63x/1.3 Glyc objective lens. Images were acquired using a sequential illumination scanning mode with the following sequences: 1) Alexa Fluor 405 signals (HyD detector/ $405 \mathrm{~nm}$ laser/415-480 nm emission range) detected together with Alexa Fluor 568 signals (HyD detector/552nm laser/560-630 nm emission range); 2) Alexa Fluor 488 signals (PMT detector/488 nm laser/ 500-550 nm emission range) detected together with Alexa Fluor 647 (PMT detector/ $638 \mathrm{~nm}$ laser / 650-750 nm emission range). Image stacks were acquired with an optimum z-step interval of $0.335 \mu \mathrm{m}$.

\section{Confocal Laser Scanning Microscopy (CLSM)}

To demonstrate the effect of LTP treatment in a short period and distance of application, we performed CLSM illustrative pictures showing the structural organization of multispecies biofilms from the negative control and the treatment with LTP for $1 \mathrm{~min}$ at $3 \mathrm{~mm}$ in a $24 \mathrm{~h}, 3$ and 7 days biofilms. Biofilms were stained with Live/Dead Bacterial Viability Kit (Baclight Bacterial Viability kit L7012; Thermo Fisher Scientific, Waltham, MA, USA) and incubated in dark at room temperature for 15 minutes to permit 
infiltration of the fluorophores inside the bacterial cells. Specimens were rinsed twice with $0.89 \% \mathrm{NaCl}$ and observed under a Leica SP8 Resonant-scanning confocal/multiphoton microscope using Leica Fluotar VISIR 25x/0.95 water objective, with a free working distance of $2.3 \mathrm{~mm}$. The pictures show live cells stained in green (Syto 9) while dead cells stained in red (Propidium lodide).

\section{Data management and statistical analyses}

The experiments were conducted in duplicate on three independent occasions $(n=6)$. The normal distribution of data was assessed by the Shapiro-Wilk test $(a=0.05)$. The data were not normally distributed so nonparametric tests were used. Tests between bacteria were made using Wilcoxon Signed Rank tests, and tests between biofilms and between groups were made using Wilcoxon Rank Sum tests. A $5 \%$ significance level was used. The analyses were done in the software SAS (SAS Institute, version 9.4, Cary, NC, USA).

\section{Results}

\section{Effect of LTP-treatments in A. naes/undii on the multispecies biofilm}

In 24-h biofilms, for the LTP-treated groups, a significant reduction in A. naes/undii was observed in all exposure times and distances when compared to the NC $(p \leq 0.014)$. LTP-treatment in all exposure times and distances also significantly reduced $A$. naes/undii as compared to the positive control $A X(p \leq 0.014)$. In contrast, LTP and MZ treatments resulted in a similar reduction of $A$. naes/undii ( $p \geq 0.240$ ). The same trend was observed for the combined treatment of $A X+M Z(p \geq 0.088)$ and $\mathrm{CHX}(p \geq 0.115)$ in comparison to LTP application at any exposure time and distance. LTP-treatment in all exposure times and distances from the nozzle significantly reduced $A$. naes/undii in the biofilm as compared to their respective flow controls $(p \leq 0.050)$. There was no statistically significant difference among the different exposure times and distances to LTP in $24 \mathrm{~h}$ biofilms ( $p \geq 0.477)$.

In the 3-day-old biofilms, LTP-treatment at all exposure times and distances of application significantly reduced $A$. naes/undii when compared to the NC $(p \leq 0.017)$. The use of LTP in any tested conditions showed similar results as the use of $\mathrm{CHX}$ for reducing $A$. naes/undii in the multispecies biofilm $(p \geq 0.051)$. All LTP-treatment conditions were more effective in reducing $A$. naes/undii in the multispecies biofilms than the positive controls $\mathrm{AX}(p \leq 0.017), \mathrm{MZ}(p \leq 0.016)$, and $\mathrm{AX}+\mathrm{MZ}(p \leq 0.016)$. All LTP-treated groups showed a higher reduction of $A$. neaslundii than the flow controls ( $p \leq 0.049)$, with exception of LTP treatment for 1 minute at $10 \mathrm{~mm}(\mathrm{P} 1 / 10)$, which showed similar results to its respective flow control $(p=0.170)$. There was no statistically significant difference in the reduction of $A$. naes/undii among the different exposure times and distances to LTP in the 3-day-old biofilm ( $p \geq 0.101)$.

In 7-day-old biofilms, there was also a significant reduction in A. naes/undii in all LTP-treated groups when compared to the NC ( $\mathrm{p} \leq 0.016)$. LTP-treatment in all exposure times and distances significantly reduced A. naes/undii when compared to treatments with the positive controls with $\mathrm{AX}(p \leq 0.016), \mathrm{MZ}(p \leq 0.047)$, 
and $A X+M Z$ ( $p \leq 0.016)$. Similar outcomes were observed comparing the LTP treatments with the positive control $\mathrm{CHX}(p \geq 0.051)$. LTP-treatment in all exposure times and distances reduced $A$. naes/undii in the biofilm compared to their respective flow controls $(p \leq 0.046)$, except for the application for 1 min and 10 $\mathrm{mm}(\mathrm{p}=0.107)$. There was no statistically significant difference in the reduction of $A$. naes/undii among the different exposure times and distances to LTP in the 7-day-old biofilm ( $p \geq 0.203)$. Figures 3 and 4 illustrate these results.

\section{Effect of LTP-treatments in P. gingivalis on the multispecies biofilm}

In 24-h biofilms, LTP treatment at all exposure times and distances of application significantly reduced $P$. gingivalis when compared to the NC $(p \leq 0.016)$. The LTP treatment at a distance of $3 \mathrm{~mm}$ for 1,3 , and 5 minutes (P1/3, P3/3, P5/3), and LTP treatment for $5 \mathrm{~min}$ at $10 \mathrm{~mm}(\mathrm{P} 5 / 10)$ significantly reduced $P$. gingivalis as compared to the positive control of $\mathrm{CHX}$ treatment $(p \leq 0.048)$. This trend was observed when comparing LTP treatment to other positive controls [AX ( $p \leq 0.021), M Z(p \leq 0.016)$ and AX+MZ $(p \leq 0.016)$ ] and the respective flow controls ( $p \leq 0.041)$, except for $\mathrm{P} 1 / 10$ group $(p=0.115)$ and $\mathrm{P} 3 / 10$ group $(p=0.149)$. There was no statistically significant difference in the reduction of $P$. gingivalis among the different exposure times and distances to LTP in the $24 \mathrm{~h}$ biofilm $(p \geq 0.203)$.

In the 3-day-old biofilms, LTP-treated groups at all exposure times and distances of application significantly reduced $P$. gingivalis in comparison to the NC $(p \leq 0.017)$. The treatment with LTP for 1 min at a distance of 3 or $10 \mathrm{~mm}$, and for $3 \mathrm{~min}$ at a distance of $10 \mathrm{~mm}$ worked as efficiently as CHX ( $\mathrm{p} \geq 0.083)$, while for the other times and distances the comparison with $\mathrm{CHX}$ showed a higher reduction of $P$. gingivalis with $\mathrm{CHX}$ treatment $(p=0.025)$. In contrast, all LTP treatments were more effective in reducing $P$. gingivalis in the multispecies biofilms than the other positive controls of $\mathrm{AX}(p \leq 0.017), \mathrm{MZ}(p \leq 0.017)$, and $A X+M Z$ ( $p \leq 0.017)$. All LTP-treated groups showed a higher reduction of $P$. gingivalis than their flow controls $(p \leq 0.049)$, with exception of P1/10, which showed similar results to its respective flow control $(p=0.282)$.

In the 7-day old biofilms, all LTP-treated groups significantly reduced $P$. gingivalis when compared to the NC $(p \leq 0.016)$. LTP-treated groups were significantly more effective in reducing $P$. gingivalis in the multispecies biofilm than the positive controls of $\mathrm{AX}(p \leq 0.016), \mathrm{MZ}(p \leq 0.016)$, and $\mathrm{AX}+\mathrm{MZ}(p \leq 0.016)$. In contrast, LTP-treated groups showed similar results to the CHX-treated groups $(p \geq 0.051)$. LTP application for $1 \mathrm{~min}$ at a distance of $3 \mathrm{~mm}(\mathrm{P} 1 / 3)$ and $10 \mathrm{~mm}$ (P1/10) significantly reduced $P$. gingivalis in the multispecies biofilm when compared to their flow controls (F1/3 and F1/10) $(p=0.025$ and $p=0.041$, respectively). There were no statistically significant differences among LTP treatments in the 7-day-old biofilm ( $p \geq 0.101)$. Figures 3 and 4 show these results. 


\section{Effect of LTP-treatments in S. oralis on the multispecies biofilm}

For the 24-h biofilm, LTP treatment at all exposure times and distances significantly reduced $S$. oralis when compared to the NC $(p \leq 0.016)$. Likewise, LTP-treatments significantly reduced $S$. oralis in the multispecies biofilms compared to the positive controls AX ( $p \leq 0.021), M Z(p \leq 0.016)$, and AX+MZ $(p \leq 0.016)$. Similar results in $S$. oralis reduction were observed for LTP and CHX-treated groups $(p \geq 0.102)$. LTP application for $5 \mathrm{~min}$ at $3 \mathrm{~mm}$ (P5/3) and $10 \mathrm{~mm}$ (P5/10) significantly reduced $S$. oralis in comparison to their flow controls ( $\mathrm{F} 5 / 3$ and $\mathrm{F} 5 / 10 ; p=0.022$ and $p=0.019$, respectively). There was no statistically significant difference in the reduction of $S$. oralis among the different exposure times and distances to LTP in the $24 \mathrm{~h}$ biofilms $(p \geq 0.203)$.

In the 3-day-old biofilms, LTP treatment at all exposure times and distances significantly reduced $S$. oralis when compared to the NC ( $p \leq 0.017)$. The treatment with LTP for $1 \mathrm{~min}$ at a distance of $10 \mathrm{~mm}$ and for 3 min at a distance of $10 \mathrm{~mm}$ worked as efficiently as $\mathrm{CHX}(\mathrm{p} \geq 0.129)$, while the other treatment groups showed a higher reduction of $S$. oralis with $\mathrm{CHX}$ treatment $(p=0.025)$. LTP-treated groups were significantly more effective in reducing $S$. oralis in the multispecies biofilm than the positive controls $A X$ $(p \leq 0.017), M Z(p \leq 0.017)$, and $A X+M Z(p \leq 0.017)$. All LTP-treated groups showed a higher reduction of $S$. oralis than their flow controls ( $p \leq 0.025)$, with exception of $\mathrm{P} 1 / 10(p=0.223)$ and $\mathrm{P} 3 / 10(p=0.185)$. There was no statistically significant difference in the reduction of $S$. oralis among the different exposure times and distances to LTP in the 3-day old biofilms ( $p \geq 0.051)$.

For the 7 days biofilms, a significant reduction in S. oralis was observed for all the LTP-treated groups when compared to the NC ( $p \leq 0.014)$. LTP application for 1,3 , and 5 minutes at $3 \mathrm{~mm}$ was more efficient in reducing $S$. oralis than positive controls of $A X(p \leq 0.036)$ and $A X+M Z(p \leq 0.035)$. LTP-treated groups resulted in similar outcomes of the treatments with $\mathrm{CHX}(p \geq 0.203)$ and MZ $(p \geq 0.051)$. LTP applications results were all similar to their flow controls $(p \geq 0.102)$. There were no statistically significant differences in $S$. oralis reduction among LTP treatments in the 7-day-old biofilm $(p \geq 0.422)$.

\section{Effect of LTP-treatments in $V$. dispar on the multispecies biofilm}

In the 24-h biofilms, a significant reduction in $V$. dispar was observed in all the LTP treatment groups when compared to the NC $(p \leq 0.016)$. Likewise, all LTP-treatments were more efficient in reducing $V$. dispar in the multispecies biofilms than the positive controls $\mathrm{CHX}(p \leq 0.018), \mathrm{AX}(p \leq 0.021), \mathrm{MZ}$ ( $p \leq 0.016)$, and $A X+M Z$ ( $p \leq 0.028)$. LTP application for $5 \mathrm{~min}$ at a distance of $3 \mathrm{~mm}(\mathrm{P} 5 / 3)$ significantly reduced $S$. oralis in the biofilm when compared to its flow control $(F 5 / 3)(p=0.016)$. There was no statistically significant difference in the reduction of $S$. oralis among the different exposure times and distances to LTP in $24 \mathrm{~h}(p \geq 0.203)$. 
In the 3-day-old biofilms, LTP treatment at all exposure times and distances significantly reduced $V$. dispar when compared to the NC $(p \leq 0.017)$. The use of LTP in all testing conditions showed similar results in the reduction of $V$. disparabundance as observed in the $\mathrm{CHX}$ treatment group $(p \geq 0.102)$. All LTPtreatments were more effective in reducing $V$. dispar in the multispecies biofilms than the other positive controls of $\mathrm{AX}(p \leq 0.017), \mathrm{MZ}(p \leq 0.034)$, and $\mathrm{AX}+\mathrm{MZ}(p \leq 0.016) . \mathrm{P} 1 / 3$ and $\mathrm{P} 3 / 3$ showed a higher reduction of $V$. dispar when compared to their respective flow controls $(p=0.012$ and $p=0.025$, respectively), while the other LTP-treatments showed similar results to their flow controls $(p \geq 0.050)$. There were no statistically significant differences in $V$. dispar reduction among LTP treatments in the 3day old biofilms $(p \geq 0.102)$.

In the 7-day-old biofilms, LTP-treated groups significantly reduced V. dispar in comparison to the NC $(p \leq 0.012)$. All LTP treatments were also more effective in reducing $S$. oralis in the multispecies biofilms as compared to the positive controls of $A X(p \leq 0.025), M Z(p \leq 0.025)$, and $A X+M Z(p \leq 0.025)$. In contrast, LTP-treated groups showed similar results in the reduction of $V$. dispar abundance as observed in the $\mathrm{CHX}$-treated groups $(p=0.102)$. LTP applications results were all similar to their respective flow controls $(p \geq 0.050)$ in the 7-day-old biofilms. There were no statistically significant differences in $V$. dispar reduction among LTP treatments in the 7-day-old biofilms $(p=1.000)$.

\section{Comparison of LTP-treatments between $24 \mathrm{~h}, 3$, and 7 days and among each bacteria}

There were no statistically significant differences between 24 h, 3 , and 7 days within each LTP treatment, or among the bacteria within each LTP treatment $(p \geq 0.250)$.

\section{Fluorescence in situ hybridization (FISH)}

FISH images confirm the presence and the distribution of $A$. naes/undii (stained in green), P. gingivalis (stained in red), S. oralis (stained in blue), and $V$. dispar (stained in yellow) in the NC biofilms up to 7 days (Figure 5).

\section{Confocal Laser Scanning Microscopy (CLSM)}

To demonstrate the effect of LTP treatment in a short period and distance, Figure 6 depicts CLSM images showing the morphology and structural organization of multispecies biofilm before and after the treatment with LTP for $1 \mathrm{~min}$ at $3 \mathrm{~mm}$ (P1/3). The images confirm the CFU results obtained, showing a lower number of live cells (stained in green) in contrast to the increase in the number of dead cells (stained in red) in biofilms formed over 24 h, 3 , and 7 days.

\section{Discussion}

Bacterial cells within a multispecies biofilm exhibit improved resistance to environmental surroundings, for instance, oxidative stress, antibiotic treatment, and nutrient depletion when compared to planktonic cells. Furthermore, there is increasing evidence that multispecies relations in the biofilm extracellular 
matrix increase the resistance against disinfectants in comparison with single-species biofilms [29] [30]. In addition, the more mature the biofilm is, the more prone it is to have augmented resistance to antimicrobial therapies, because of its anti-phage action, protection against monoclonal antibodies, and protection exerted by components of the extracellular matrix [31]. Considering the importance of using a polymicrobial biofilm to simulate approach peri-implantitis conditions, we applied an in vitro biofilm model that was previously validated [17], composed of four important peri-implantitis-related bacteria. This variety of microorganisms in our biofilm model was confirmed using FISH probes, a molecular biology tool that we applied to detect the presence and of $A$. naes/undii, P. gingivalis, S. oralis, and $V$. dispar in 24-h, 3- and 7-day-old biofilms.

Models to study biofilms in vitro gradually contribute to to the existing data of biofilm physiology within the host condition and can be later translated into complex in vivo models of tissues infections. In vitro models have helped address elementary questions regarding biofilm development, physiology, and architecture. They offer many advantages such as a low cost, easy set-up, and responsiveness to high throughput screens [32]. Therefore, in this study, we applied a validated model of in vitro peri-implantitisrelated biofilm to test the effects of a single LTP treatment on different stages of biofilm formation.

The present study confirmed our hypothesis that a single LTP treatment in vitro was sufficient to disrupt and disinfect in vitro multispecies peri-implantitis-related biofilm more effectively than the controls. The application of LTP at $3 \mathrm{~mm}$ starting from $1 \mathrm{~min}$ demonstrated to be an effective measure in reducing the multispecies biofilm formed over $24 \mathrm{~h}, 3$, and 7 days by $A$. naes/undii, P. gingivalis, $S$. oralis, and $V$. dispar on titanium surfaces. The application of LTP for 5 min demonstrated to be effective at both distances of $3 \mathrm{~mm}$ and $10 \mathrm{~mm}$. Therefore, time of application and distance influenced the outcome of LTP treatments. With a shorter distance $(3 \mathrm{~mm})$ and the shortest period of application (1 $\mathrm{min})$, LTP application was enough to reduce or kill bacteria in any of the tested periods of biofilm formation. With a longer distance $(10 \mathrm{~mm})$, the effectiveness of LTP depended on a longer period of application to obtain similar results as LTP $1 \mathrm{~min}$ at $3 \mathrm{~mm}$. A previous study by Nicol et al. [21] showed a similar trend in results where the increase in distance between the jet nozzle and sample, as well as decreased voltage and flow rate, led to a decreased efficacy of LTP treatment. It is speculated that the addition of oxygen with longer distances between the sample and the LTP nozzle can lead to decreasing plume length, electron density, and ionization [33]. In contrast to time and distance of application, the age of the biofilms did not show to influence the LTP effects.

These results are remarkable since it is a short period of LTP application resulting in very few or zero bacterial recoveries. This was also confirmed through live and dead confocal images, which clearly showed that the treatment for $1 \mathrm{~min}$ at $3 \mathrm{~mm}$ with LTP killed bacteria on the titanium surfaces. It shows that LTP is penetrating and killing the bacteria within the biofilm. In contrast to current peri-implantitis treatment strategies, previous studies demonstrated that LTP has the unique potential to concurrently decontaminate and/or detoxify biofilm infected surfaces while creating a suitable environment for bone regrowth around the implant without harming peri-implant healthy host tissue [22] [23] [26] [27]. 
Advantages of LTP over traditional antimicrobial applications are that LTP can be used for site-specific treatment; and provides a quick bactericidal response leading to a potential decrease in the risk of developing antimicrobial resistance due to LTP's multiple modes of action and diversity of active agents [21], and there are minimal side effects [21]. Antibiotic resistance is problematic at the therapeutic level in health sciences all over the world. As stated by the Centers for Disease Control, about 2.8 million antibiotic-resistant bacterial infections occur in the United States and cause more than 35,000 deaths annually [34]. The use of antimicrobials as assistants to periodontal mechanical therapy has been debatable and is frequently kept for cases of aggravation of periodontal lesions or in patients who do not respond immediately to conventional mechanical therapy or in patients who do not respond instantly respond to conventional mechanical therapy or in individuals who are in danger of having systemic compromise [35]. Metronidazole and amoxicillin are the main assistant treatment to prevent or decrease periodontal pathology development [36]; however, it has been reported a high number of periodontal disease-related bacteria that are resistant to these antibiotics [37] [35]. In fact, we observed in our study that both metronidazole and amoxicillin were not effective in reducing the viability of the tested periimplantitis-related biofilms in vitro. Therefore, it is of high importance to look for possible treatments to avoid bacterial resistance, in this context, LTP is a great candidate as a coadjuvant or alternative therapy for peri-implantitis treatment.

Tissue-compatible temperatures of plasma encourage LTP's use in vivo [22] [23]. Recently, immediate and post-recovery cellular-level responses to LTP (helium-based) were examined in vitro using human gingival fibroblasts and osteoblasts (MC3T3-E1 cells) and in vivo in rat calvarial bone and the adjacent periosteum. The results showed that a direct LTP treatment for up to 3 min caused no harmful effects at either the cellular or tissue levels [38].

Dental implants previously treated with LTP showed greater bone-to-implant contact ratio, inter-thread, and peri-implant bone density [39]. Physicochemical and in vivo results demonstrated that air based LTP treatment augmented surface energy and supported earlier osseointegration as compared to the controls [26]. Hence, LTP can potentially act as a coadjuvant to flap surgery in efficiently treating peri-implantitis, a necessary first step toward the development of novel efficient treatments to increase the longevity and success rate of millions of implants currently in function. LTP, coupled with surgical debridement, might be a unique efficacious approach for treating peri-implantitis by reducing biofilm viability, increasing the levels of peri-implant tissue re-attachment, and enhancing implant surface characteristics for bone tissue healing.

LTP antibiofilm activity against a multispecies mature biofilm can go beyond the oral cavity, being a potential candidate as a safe antimicrobial co-adjuvant for other types of implants. For example, the use of arthroplasty enables the replacement of a natural joint with artificial material or a joint prosthesis. Even though arthroplasty is very effective and has enhanced the quality of life of many patients, implantrelated problems can happen throughout the lifetime [40]. The consequences of biofilms in treatment and results of joint prostheses are massive, as they confer phenotypical resistance that may require new surgeries and extended treatments. Consequently, it is of greatest relevance to evade bacterial 
colonization of implants, preventing infection [40]. In this context, the use of LTP in surgical implants to deliver its therapeutic effects to patients is encouraged, since there is a continued determination to improve the clinical performance of surgical implants, aiming the implant-tissue interface [41]. As a multipurpose and powerful tool, LTP is capable of upgrading surgical implants using various strategies of interface biotechnology, for instance, surface modification, coating deposition, and drug delivery [41], in addition to being an excellent antibacterial, as confirmed by this study.

LTP represents an innovative technology for the treatment of peri-implantitis. No other treatment has been shown to have this unique combination of therapeutic effects. We conclude that the LTP application is a promising treatment to disinfect the peri-implant surroundings, which can potentially act as a coadjuvant therapy during the surgical procedure. Furthermore, LTP is effective against biofilms at a short distance of $3 \mathrm{~mm}$ starting from a short exposure time of $1 \mathrm{~min}$. The present study established parameters that will be further tested in a more complex in vivo model.

\section{Declarations}

\section{Funding}

This work was supported by the National Institute of Dental and Craniofacial Research (NIH/NIDCR 1R21DE028929-01).

\section{Author Contributions}

B.H.D.P carried out all the experiments and wrote the main manuscript, D.P.M helped B.H.D.P in the execution of the experiments. G.J.E. made the statistics and contributed to the interpretation of the results. P.G.C and S.D conceptualized the study, led the project, and provided resources. All authors provided critical feedback and helped shape the research, analysis, and manuscript.

\section{Conflict of Interest}

The authors declare no competing interests.

\section{References}

1. Dabdoub, S. \& Tsigarida, A. K., P. Patient-specific analysis of periodontal and peri-implant microbiomes. J Dent Res. 92, 168S-75S (2013).

2. Tarnow D. Increasing Prevalence of Peri-implantitis: How Will We Manage?. J Dent Res. 95, 7-8 (2016).

3. Claffey, N., Clarke E. Polyzois I. \& Renvert S. Surgical treatment of peri-implantitis. J Clin Periodontol. 35, 316-332 (2008).

4. Caton, J. Armitage, G., Berglundh, T., Chapple, I., Jepsen, S., Kornman, K., et al. B. A new classification scheme for periodontal and peri-implant diseases and conditions- Introduction and key changes 
from the 1999 classification. J Clin Periodontol. 45, S1-S8 (2018).

5. Muñoz, V., Duque, A., Giraldo, A. \& Manrique, R. Prevalence of Peri-implant Disease According to Periodontal Probing Depth and Bleeding on Probing: A Systematic Review and Meta-Analysis. Int J Oral Maxillofac Implants. 33(4), e89-e105 (2018).

6. Shibli, J., Melo, L., Ferrari, D., Figueiredo, L., Faveri, M. \& Feres,M. Composition of supra- and subgingival biofilm of subjects with healthy and diseased implants. Clin Oral Implants Res. 19, 975982 (2008).

7. Socransky,S., Haffajee, A., Cugini, M., Smith, C \& Kent, R.J. Microbial complexes in subgingival plaque. J Clin Periodontol. 25, 134-144 (1998).

8. Nyvad, B. \& Kilian,M. Microbiology of the early colonization of human enamel and root surfaces in vivo. Scand J Dent Res. 95, 369-380 (1987).

9. Li, J., Helmerhorst, E., Leone, C., Troxler, R., Yaskell, T., Haffajee A., et al.. Identification of early microbial colonizers in human dental biofilm. J Appl Microbiol. 97,1311-1318 (2004).

10. Diaz, P., Chalmers, N., Rickard, A., Kong, C., Milburn, C., Palmer R.J., et al. Molecular characterization of subject-specific oral microflora during initial colonization of enamel. Appl Environ Microbiol, 72, 2837-2848 (2006).

11. Kolenbrander, P., Palmer, R. J., Rickard, A., Jakubovics, N., Chalmers, N. \& Diaz, P. Bacterial interactions and successions during plaque development. Periodontol 2000. 42, 47-79 (2006)..

12. Periasamy S. \& Kolenbrander P. Central role of the early colonizer Veillonella sp. in establishing multispecies biofilm communities with initial, middle, and late colonizers of enamel. J Bacteriol. 192, 2965-2972 (2010).

13. How, K., Song K. \& Chan, K. Porphyromonas gingivalis: An Overview of Periodontopathic Pathogen below the Gum Line. Front Microbiol. 7, 53 (2016).

14. Tabanella, G., Nowzari, H. \& J. Slots, "Clinical and microbiological determinants of ailing dental implants.Clin Implant Dent Relat Res. 11, 24-36 (2009).

15. Casado, P., Otazu, I., Balduino, A., de Mello, W., Barboza, E. \& Duarte, M. Identification of periodontal pathogens in healthy periimplant sites. Implant Dent. 20, 226-235 (2011).

16. Persson,G. \& Renvert, S. Cluster of bacteria associated with peri-implantitis. Clin Implant Dent Relat Res. 16, 783-793 (2014).

17. Kommerein, N., Stumpp, S., Müsken, M., Ehlert, N., Winkel, A., Häussler, S., et al. An oral multispecies biofilm model for high content screening applications. PLoS One. 12, e0173973 (2017).

18. Valderrama, $P \&$ Wilson, T.J. Detoxification of implant surfaces affected by peri-implant disease: an overview of surgical methods. Int J Dent., 2013, 740680 (2013).

19. Rokaya, D., Srimaneepong, V., Wisitrasameewon, W., Humagain, M., \& Thunyakitpisal, P. Periimplantitis Update: Risk Indicators, Diagnosis, and Treatment. Eur J Dent. 14, 672-682 (2020).

20. Duarte, S. \& Panariello, B. H. D. Comprehensive biomedical applications of low temperature plasmas. Arch Biochem Biophys. 693,108560 (2020). 
21. Nicol, M., Brubaker, T., Honish, B., Simmons, A., Geissel, K. A. M., Whalen, C., et al. Antibacterial effects of low-temperature plasma generated by atmospheric-pressure plasma jet are mediated by reactive oxygen species. Sci Rep. 10, 3066 (2020).

22. Carreiro, A., Delben, J. A., Guedes, S., Silveira, E., Janal, M., Vergani, C., et al. Low-temperature plasma on peri-implant-related biofilm and gingival tissue. J Periodontol. 90, 5, 507-515 (2019).

23. Delben, J., Zago, C., Tyhovych, N., Duarte, S. \& Vergani, C. Effect of Atmospheric-Pressure Cold Plasma on Pathogenic Oral Biofilms and In Vitro Reconstituted Oral Epithelium. PloS one. 11, e0155427 (2016).

24. Bernhardt, T., Semmler, M., Schäfer, M., Bekeschus, S., Emmert, S. \& Boeckmann, L. Plasma medicine: applications of cold atmospheric pressure plasma in dermatology. Oxid Med Cell Longev, 2019, 3873928 (2019).

25. Mylonas, C., \& Kouretas, D. Lipid peroxidation and tissue damage. In Vivo. 13, 295-309 (1999).

26. Danna, N., Beutel, B.,Tovar, N., Witek, L., Marin, C., Bonfante, E., et al. Assessment of Atmospheric Pressure Plasma Treatment for Implant Osseointegration. Biomed Res Int. 2015, 761718 (2015).

27. Guastaldi, F., Yoo, D., Marin, C., Jimbo, R.,Tovar, N., Zanetta-Barbosa, D. \& Coelho, P. Plasma treatment maintains surface energy of the implant surface and enhances osseointegration. Int $\mathrm{J}$ Biomater. 2013,354125 (2013).

28. Giro, G., Tovar, N., Witek, L., Marin, C., Silva, N., Bonfante, E. \& Coelho, P. Osseointegration assessment of chairside argon-based nonthermal plasma-treated Ca-P coated dental implants. Biomed Mater Res A., 101, 98-103 (2013).

29. Lee, K., Periasamy, S., Mukherjee, M., Xie, C., Kjelleberg, S. \& Rice, S. Biofilm development and enhanced stress resistance of a model, mixed-species community biofilm. ISME J. 8, 894-907 (2014).

30. Sanchez-Vizuete, P., Orgaz, B., Aymerich, S., Le Coq, D. \& Briandet, R. Pathogens protection against the action of disinfectants in multispecies biofilms. Front microbiol. 6, 705 (2015).

31. L. Zhang, E. Liang, Y. M. T. Cheng, F. Ge, K. Zhou, M. Bao, L. Lv, L. Li, J. Yi, C. Lu and Y. Tan, "Is combined medication with natural medicine a promising therapy for bacterial biofilm infection?," Biomedicine \& Pharmacotherapy, vol. 128, p. 110184, 2020.

32. D. Lebeaux, A. Chauhan, O. Rendueles and C. Beloin, "From in vitro to in vivo Models of Bacterial Biofilm-Related Infections.," Pathogens (Basel, Switzerland), vol. 2, no. 2, p. 288-356, 2013.

33. H. M. Joh, J. Y. Choi, S. J. Kim, T. H. Chung and T. H. Kang, "Effect of additive oxygen gas on cellular response of lung cancer cells induced by atmospheric pressure helium plasma jet.," Science Reports, vol. 4, 2014.

34. CDC. Antibiotic Resistance Threats in the United States. Control and Prevention (U.S.): Atlanta, GA, USA (2019).

35. Aragón, N., Jaramillo-Echeverry, A. \& Ramirez-Malule, H. Bibliometric analysis of bacterial resistance on periodontal disease," Journal of Applied Pharmaceutical Science. 11, 118-124 (2021). 
36. McGowan, K., McGowan, T. \& Ivanovski, S. Optimal dose and duration of amoxicillin-plusmetronidazole as an adjunct to non-surgical periodontal therapy: a systematic review and metaanalysis of randomized, placebo-controlled trials. J Clin Periodontol. 45, 56-67 (2018).

37. Ardila, C., Granada, M. \& Guzmán, I. Antibiotic resistance of subgingival species in chronic periodontitis patients. J Periodontal Res. 45, 557-563 (2010).

38. Lee, J., Park, S., Kim, K., Yoon, S., Kim, G., Lee, Y. \& Seol, Y. J. Safety evaluation of atmospheric pressure plasma jets in in vitro and in vivo experiments. J Periodontal Implant Sci. 51, 213-223 (2021).

39. Naujokat, H., Harder, S., Schulz, L., Wiltfang, J., Flörke, C. \&. Açil, Y. J. Surface conditioning with cold argon plasma and its effect on the osseointegration of dental implants in miniature pigs. J. CranioMaxillo-Fac. Surg. 47, 484-490 (2019).

40. Esteban, J., Vallet-Regí, M. \& Aguilera-Correa, J. Antibiotics- and Heavy Metals-Based Titanium Alloy Surface Modifications for Local Prosthetic Joint Infections. Antibiotics, 10, 1270 (2021).

41. Tan, F., Fang, Y., Zhu, L., \& Al-Rubeai, M. Cold atmospheric plasma as an interface biotechnology for enhancing surgical implants. Crit Rev Biotechnol, 41, 425-440 (2021).

\section{Figures}




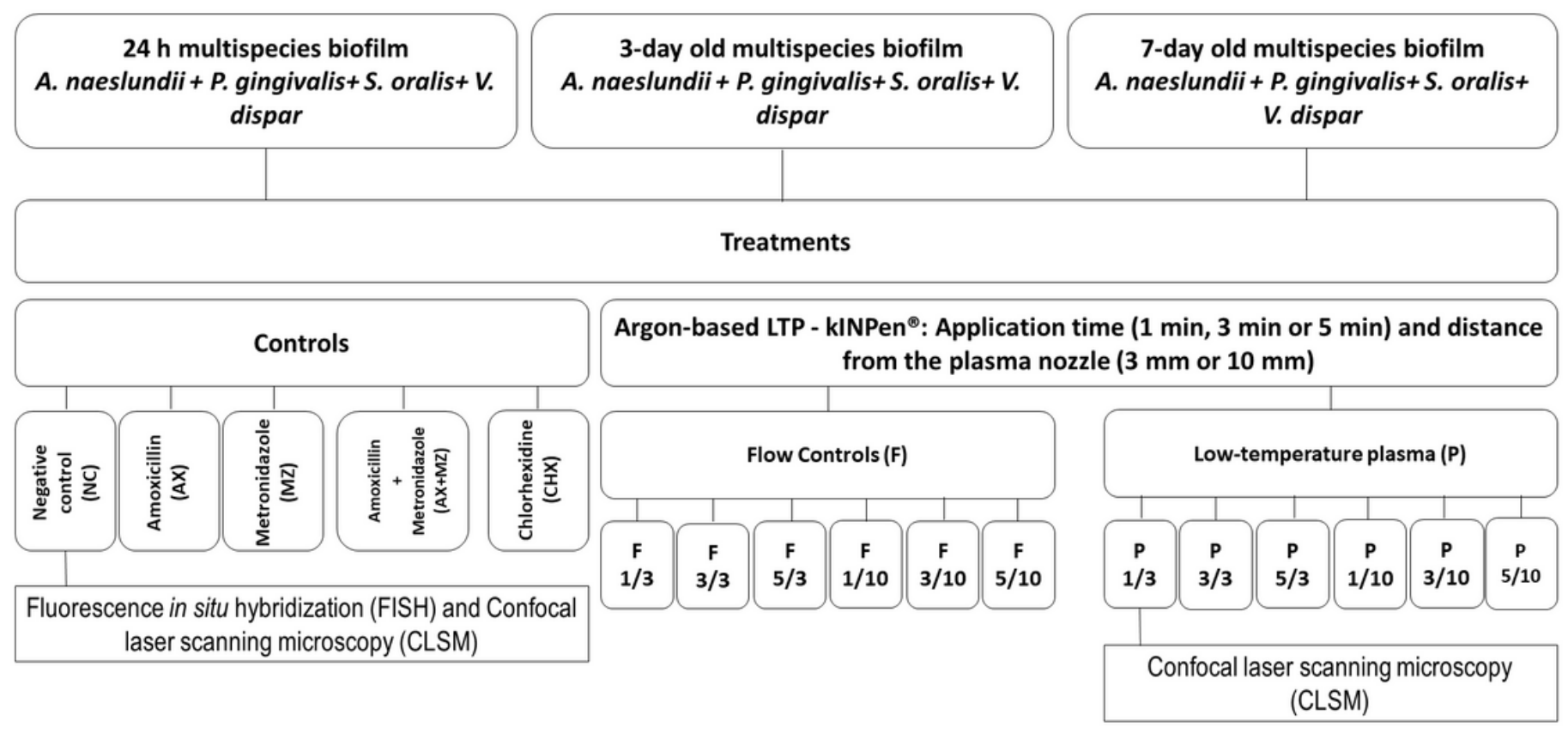

Figure 1

Flowchart representing the study design. 


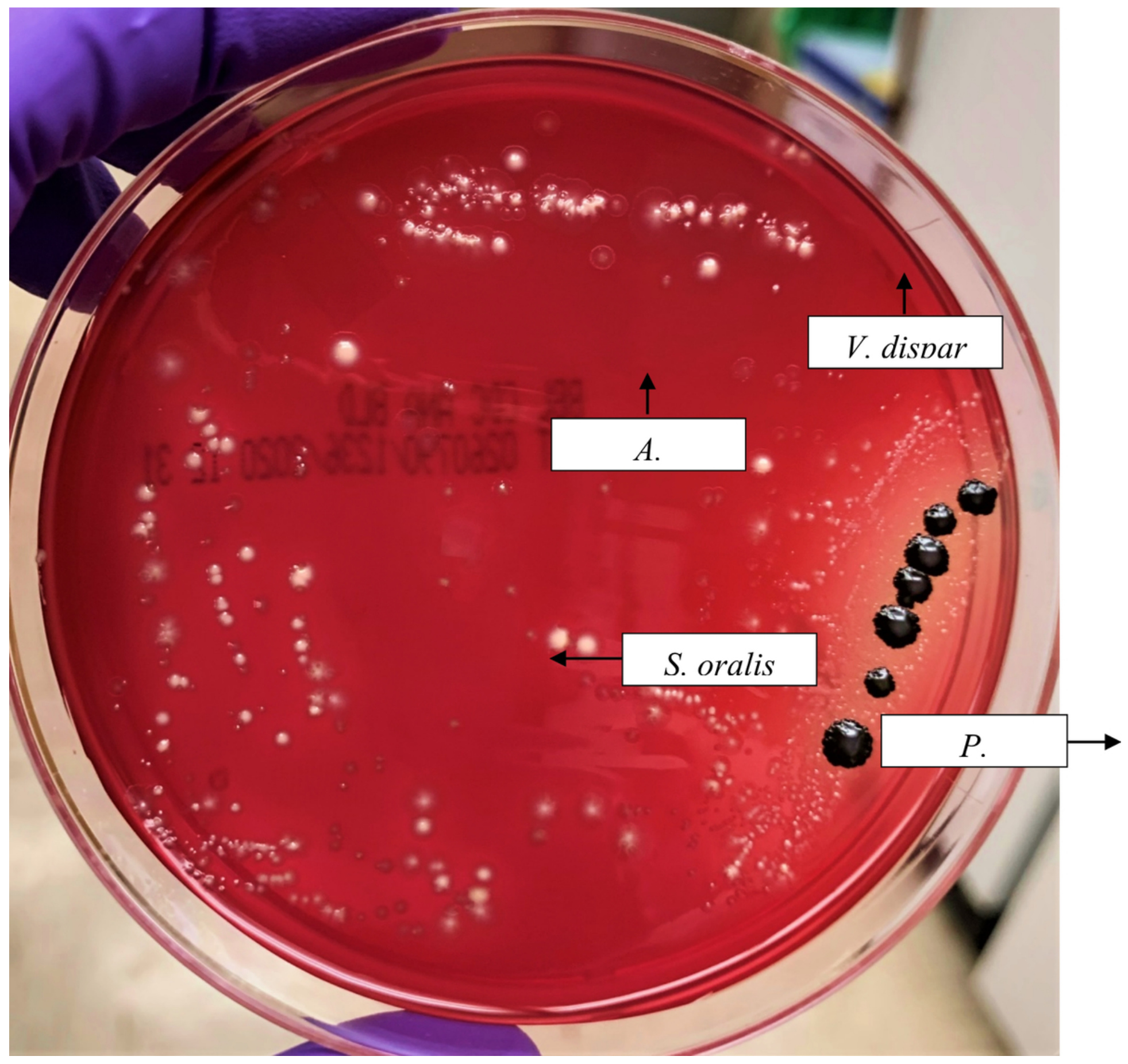

Figure 2

Four-species biofilm growth on blood agar plates. A. naeslundii show crème/red-pigmented round colonies without hemolytic halo, P. gingivalis present black-pigmented round colonies with a hemolysis halo, S. oralis grows characteristically in chains and forms small white colonies and V. dispar colonies show a transparent halo with a white spot in the middle. 


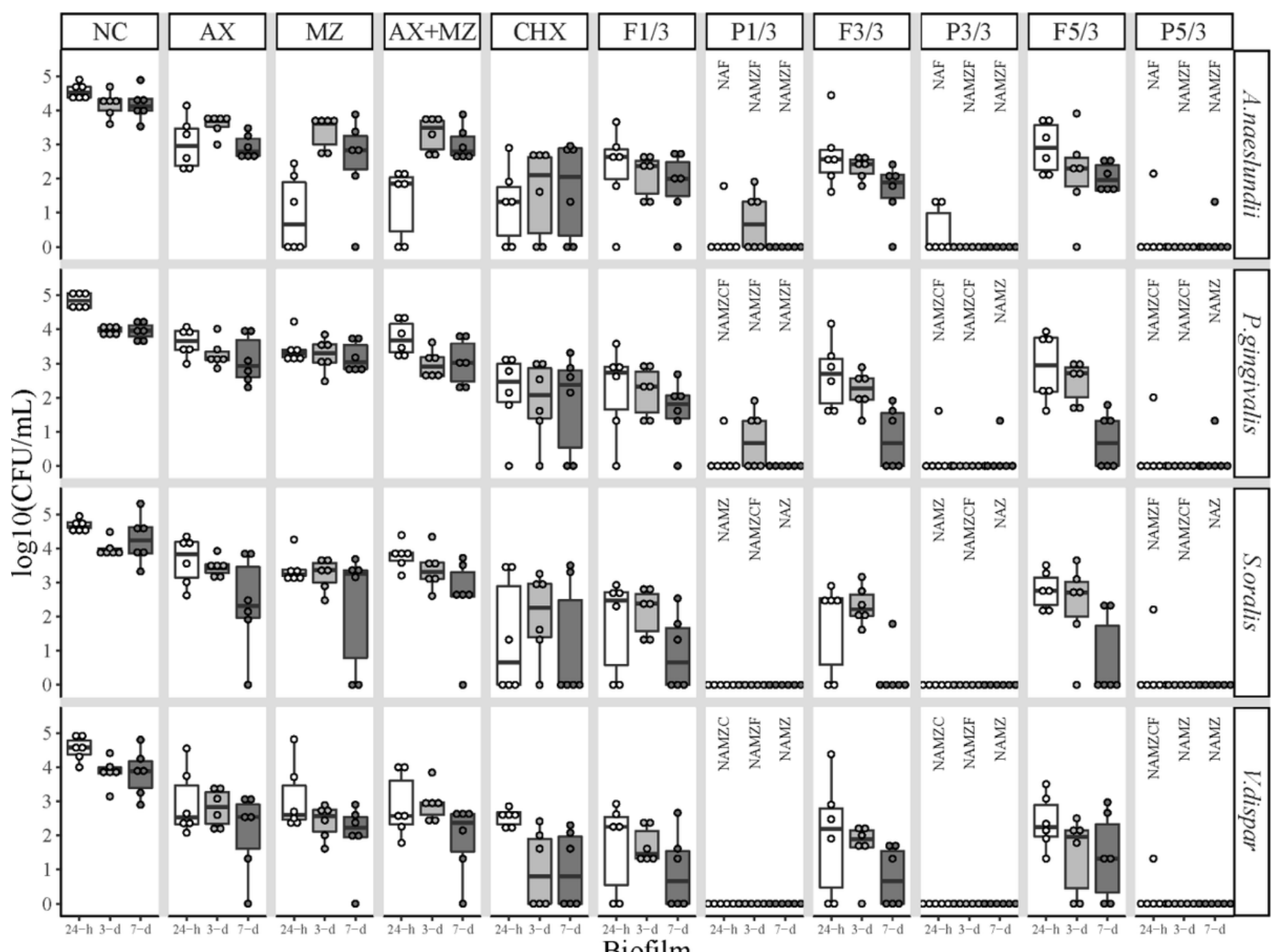

Figure 3

Box-plot of $\log 10(\mathrm{CFU} / \mathrm{mL})$ of $24 \mathrm{~h}, 3$, and 7 days multispecies biofilms composed by A. naeslundii, $\mathrm{P}$. gingivalis, S. oralis, and V. dispar at a distance of $3 \mathrm{~mm}$ from the equipment nozzle. NC stands for the negative control, which are the biofilms that received no treatment. P stands for LTP-treated groups for 1 , 3 , or 5 min $(P 1 / 3, P 3 / 3, P 5 / 3)$, Flow $(F)$ is the control for the LTP treatments $(F 1 / 3, F 3 / 3, F 5 / 3)$. As positive controls, samples were treated with $0.12 \%$ chlorhexidine (CHX), Amoxicillin (AX), Metronizadole $(M Z)$, and amoxicillin combined with Metronidazole $(A X+M Z)$. The letters represent statistical significance above each of the plasma treatments $(n=6 ; p \leq 0.05$; Wilcoxon Signed Rank and Wilcoxon Rank Sum tests). Letter $\mathrm{N}$ is for significance versus $\mathrm{NC}$, letter $\mathrm{A}$ is for significance vs $\mathrm{AX}$, letter $\mathrm{M}$ is for significance vs $M Z$, letter $Z$ is for significance vs $A X+M Z$, letter $C$ is for significance vs $C H X$, and letter $F$ is for significance vs its corresponding flow control. There were no statistically significant differences between the plasma treatments, between $24 \mathrm{~h}$ and $7 \mathrm{~d}$ within each plasma treatment, or between the bacteria within each plasma treatment. 


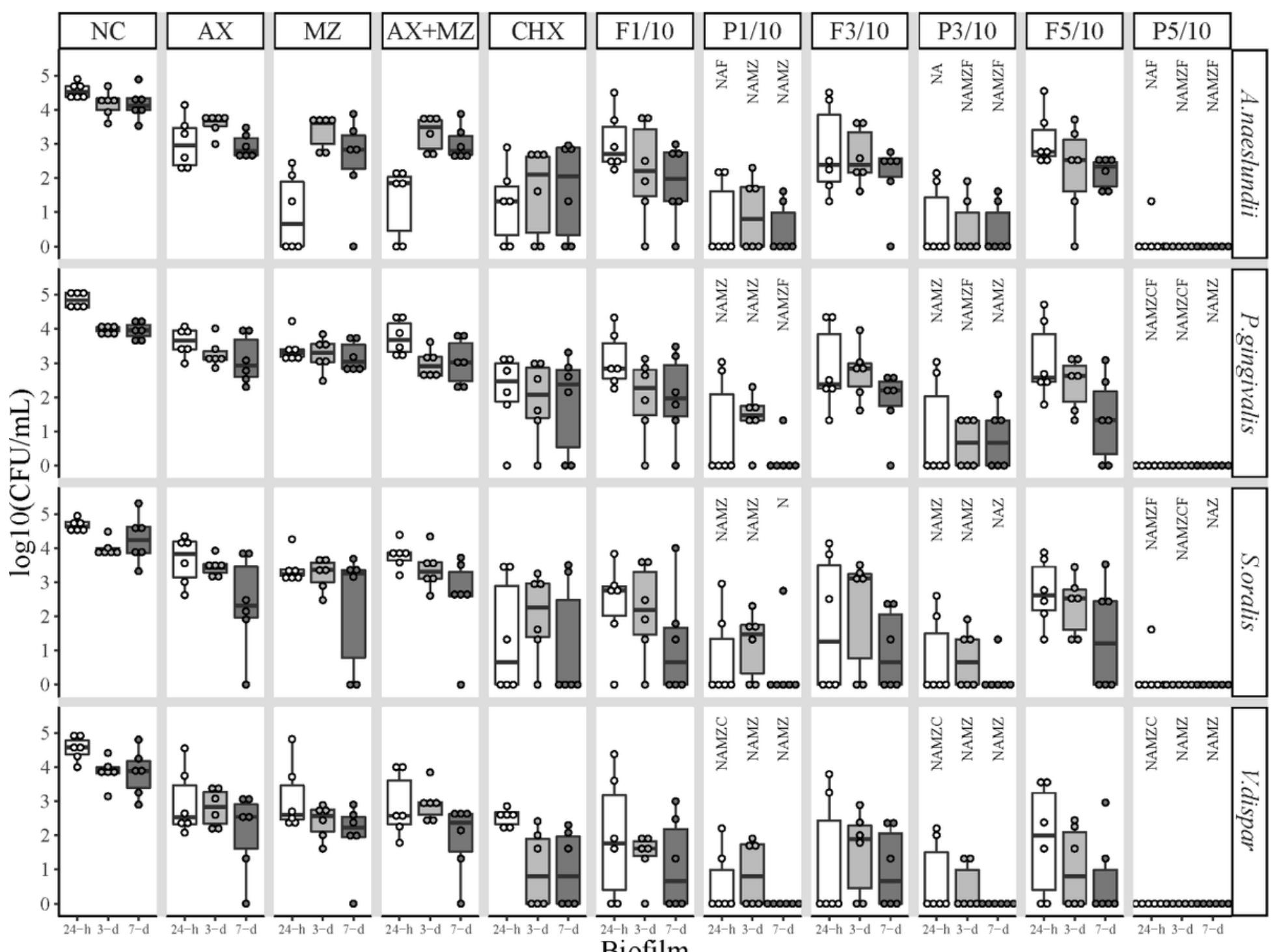

Figure 4

Box-plot of $\log 10(\mathrm{CFU} / \mathrm{mL})$ of $24 \mathrm{~h}, 3 \mathrm{~d}$, and 7 days multispecies biofilms composed by A. naeslundii, P. gingivalis, S. oralis, and V. dispar at a distance of $10 \mathrm{~mm}$ from the equipment nozzle. NC stands for the negative control, which are the biofilms that received no treatment. P stands for LTP-treated groups for 1 , 3 , or 5 min (P1/10, P3/10, P5/10), Flow (F) is the control for the LTP treatments (F1/10, F3/10, F5/10). As positive controls, samples were treated with $0.12 \%$ chlorhexidine (CHX), Amoxicillin (AX), Metronizadole $(M Z)$, and amoxicillin combined with Metronidazole $(A X+M Z)$. The letters represent statistical significance above each of the plasma treatments $(n=6 ; p \leq 0.05$; Wilcoxon Signed Rank and Wilcoxon Rank Sum tests). Letter $\mathrm{N}$ is for significance versus $\mathrm{NC}$, letter $\mathrm{A}$ is for significance vs $\mathrm{AX}$, letter $\mathrm{M}$ is for significance vs $M Z$, letter $Z$ is for significance vs $A X+M Z$, letter $C$ is for significance vs $C H X$, and letter $F$ is for significance vs its corresponding flow control. There were no statistically significant differences between the plasma treatments, between $24 \mathrm{~h}$ and $7 \mathrm{~d}$ within each plasma treatment, or between the bacteria within each plasma treatment. 


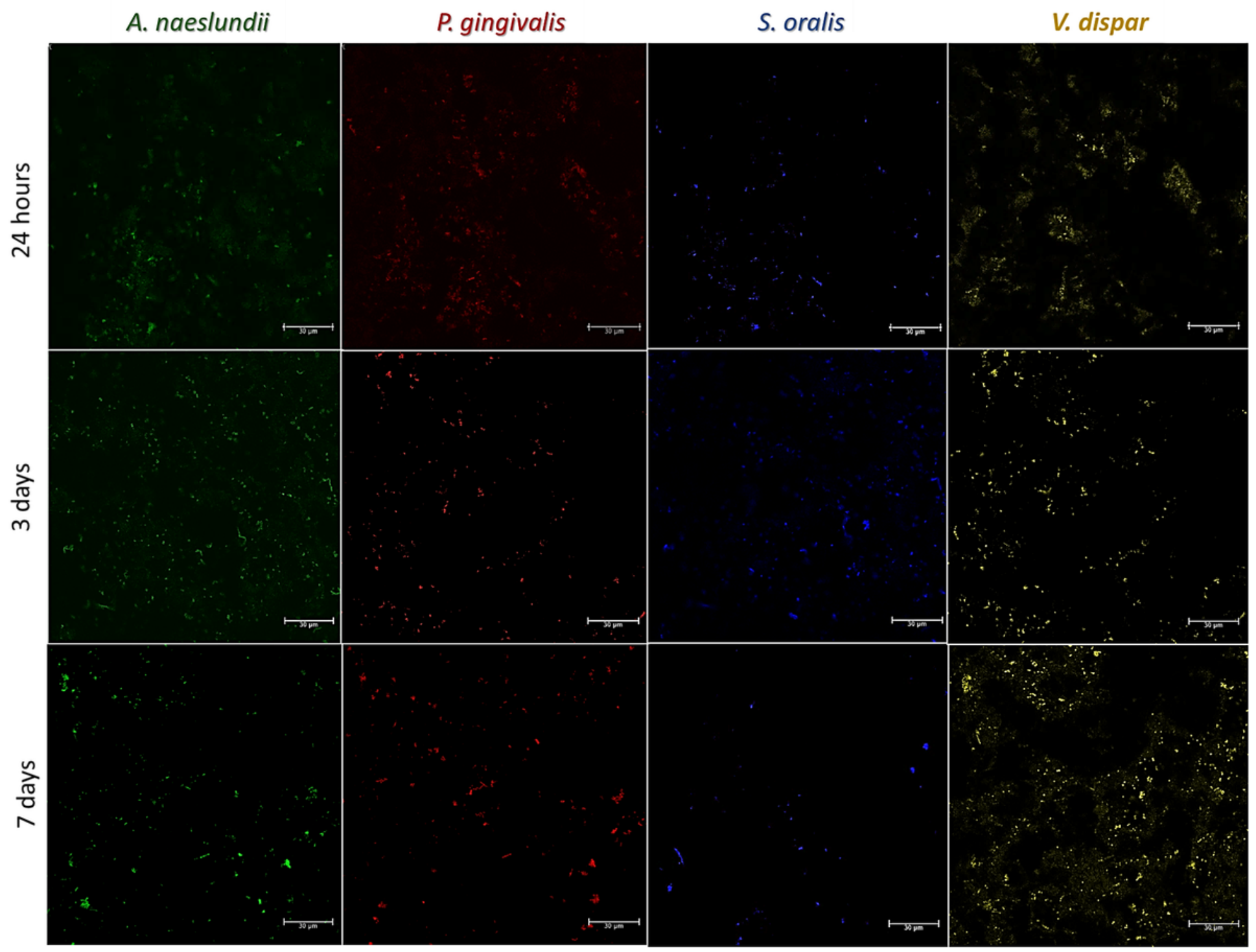

Figure 5

$24 \mathrm{~h}, 3$, and 7 days multispecies biofilms stained with FISH probes for A. naeslundii (Alexa Fluor-488; green), P. gingivalis (Alexa Fluor-647; red), S. oralis (Alexa Fluor-405; blue), and V. dispar (Alexa Fluor-568; yellow). 

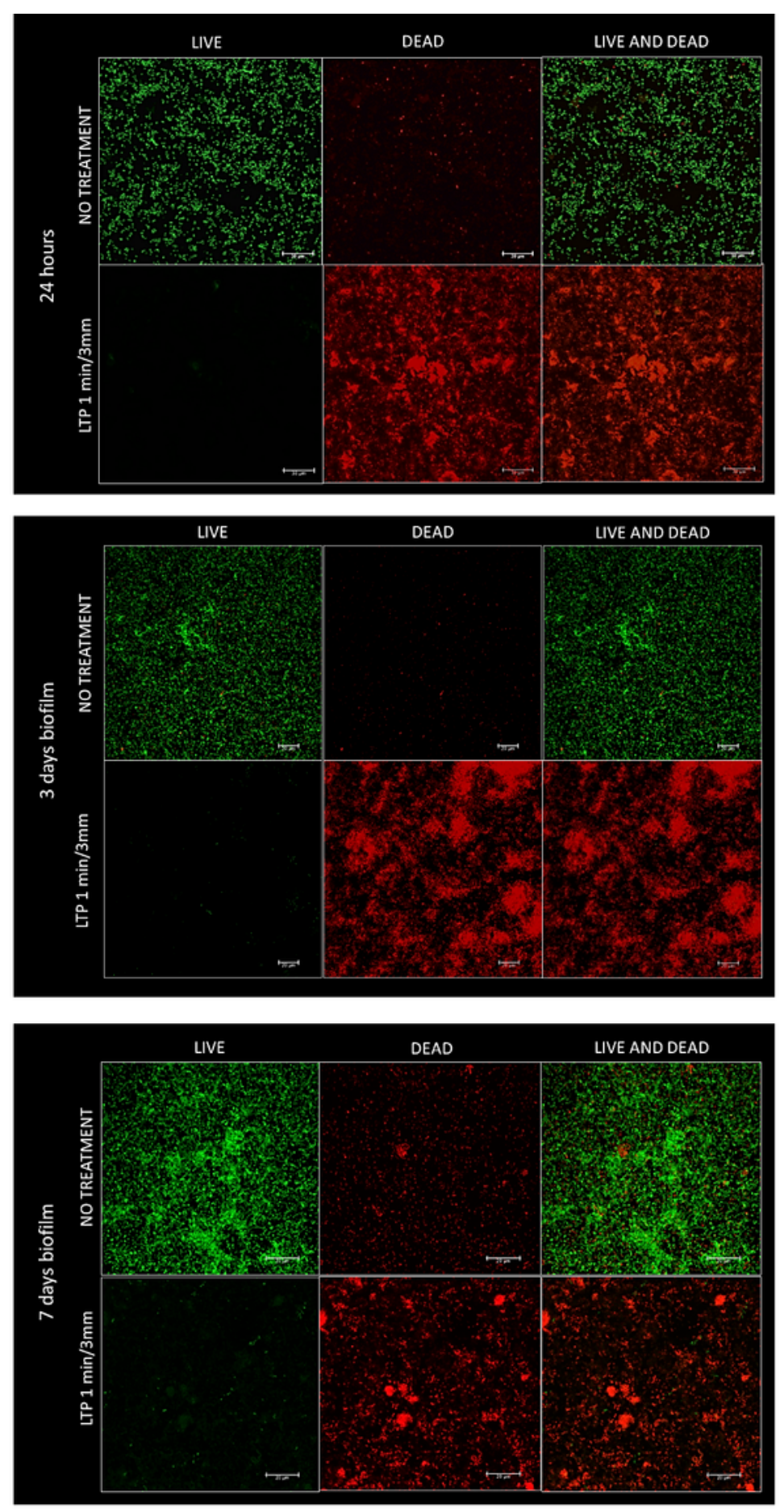

Figure 6

CLSM images of $24 \mathrm{~h}, 3$, and 7 days multispecies biofilms. The images show live cells that are stained green (Syto 9) while dead cells are stained red (Propidium lodine). 